\title{
The impact of early-life exposure to food allergens on the subsequent development of sensitisation and allergy to foods
}

\author{
R. L. Thompson ${ }^{1}$, L. M. Miles ${ }^{1}$, J. Lunn ${ }^{1}$, G. Devereux ${ }^{2}$, J. Strid $^{3}$ and J. L. Buttriss ${ }^{1}$ \\ ${ }^{1}$ British Nutrition Foundation, High Holborn House, 52-54 High Holborn, London WCIV 6RQ, UK, ${ }^{2}$ Department \\ of Environmental and Occupational Medicine, University of Aberdeen, Foresterhill, Aberdeen, AB25 2ZD, UK and \\ ${ }^{3}$ Peter Gorer Department of Immunobiology, King's College London School of Medicine at Guy's Hospital, London, \\ SE1 9RT, UK
}

\begin{abstract}
The present systematic review aimed to evaluate the influence of early-life exposure (maternal and childhood) to foods and the subsequent development of sensitisation or allergy to food during childhood. Studies published from 1 January 1999 to 7 March 2008 were identified using electronic databases and bibliography searches. Human studies that assessed the impact of non-avoidance compared with avoidance or reduced quantities of foods on either food sensitisation or allergy, or both outcomes, were eligible.

Seven studies investigating maternal exposure to food allergens and development of food sensitisation and allergy in the child were identified: one non-randomised clustered trial, two cohort studies and four case-control studies. These studies were mostly of poor quality and a heterogeneous range of exposures were studied. Overall, there was no association between maternal diet during pregnancy or lactation and food sensitisation and allergy in the child. In total nineteen studies (eight trials, ten cohort studies and one case-control study) investigated whether dietary consumption of food allergens, or their avoidance, in childhood had any impact on subsequent development of food sensitisation or allergy. Six studies (five trials and one cohort) reported on infants fed cow's milk formula compared with breast milk, but there was no consistent evidence of an association between infants receiving cow's milk formula and the development of subsequent food sensitisation or allergy in the child. Evidence from eight studies (mostly cohorts) did not show that increased duration of breast-feeding protects against food allergy. Four cohort studies and one case-control study investigated the effects of timing of introduction of solid food on subsequent food allergy and sensitisation; there was no consistent evidence either in general or for specific foods to support an association. Three randomised controlled trials investigated multifaceted interventions consisting of avoidance of multiple allergenic foods as well as household measures (such as house dust mite (Dermatophagoides spp.) avoidance) in high-risk populations. These studies provided some evidence to show that such multifaceted interventions aimed at mothers and infants protect against food sensitisation (but not food allergy) in the child.

Overall, the heterogeneous nature of the evidence, and lack of high-quality studies, has hindered the development of definitive conclusions. Nevertheless, the available evidence does not indicate that either maternal exposure, or early or delayed introduction of allergenic foods in the diets of children, impacts on subsequent development of food sensitisation or allergy.
\end{abstract}

